\title{
New Guidance for Using t-SNE: Alternative Defaults, Hyperparameter Selection Automation, and Comparative Evaluation
}

\author{
Robert Gove, Lucas Cadalzo, Nicholas Leiby, Jedediah M. Singer, Alexander \\ Zaitzeff \\ Two Six Technologies
}

\begin{abstract}
We present new guidelines for choosing hyperparameters for t-SNE and an evaluation comparing these guidelines to current ones. These guidelines include a proposed empirically optimum guideline derived from a t-SNE hyperparameter grid search over a large collection of data sets. We also introduce a new method to featurize data sets using graph-based metrics called scagnostics; we use these features to train a neural network that predicts optimal t-SNE hyperparameters for the respective data set. This neural network has the potential to simplify the use of t-SNE by removing guesswork about which hyperparameters will produce the best embedding. We evaluate and compare our neural network-derived and empirically optimum hyperparameters to several other t-SNE hyperparameter guidelines from the literature on 68 data sets. The hyperparameters predicted by our neural network yield embeddings with similar accuracy as the best current t-SNE guidelines. Using our empirically optimum hyperparameters is simpler than following previously published guidelines but yields more accurate embeddings, in some cases by a statistically significant margin. We find that the useful ranges for t-SNE hyperparameters are narrower and include smaller values than previously reported in the literature. Importantly, we also quantify the poten-
\end{abstract}

Email address:

\{robert.gove|lucas.cadalzo|nick.leibyljed.singer|alexander.zaitzeff\}@twosixtech.com (Robert Gove, Lucas Cadalzo, Nicholas Leiby, Jedediah M. Singer, Alexander Zaitzeff)

Preprint submitted to Visualization Meets AI

March 21, 2022 
tial for future improvements in this area: using data from a grid search of t-SNE hyperparameters we find that an optimal selection method could improve embedding accuracy by up to two percentage points over the methods examined in this paper.

Keywords: Dimensionality reduction, machine learning, t-SNE

2010 MSC: 00-01, 99-00

\section{Introduction}

Data analysts use t-SNE [1] and related dimensionality reduction methods to explore large, high-dimensional data sets. These methods produce an embedding in a low-dimensional space, usually two or three dimensions. These embeddings

5 are then visualized to analyze clusters, outliers, and relationships between the points in the data set. Here we describe and analyze new and existing guidelines for using t-SNE. We perform an empirical comparison of t-SNE hyperparameters across hundreds of data sets, and create a machine learning model to predict the accuracy of a data set's embedding given some easily calculated structural characteristics.

t-SNE has hyperparameters that influence the resulting embedding. Software packages like Scikit-learn [2] and OpenTSNE [3] provide default hyperparameters so that users are not required to specify them each time they use t-SNE. However, the fact that these hyperparameters can be specified by users presumably reflects the belief that there are no default settings that will work well on all data sets. Some prior studies have claimed that t-SNE is robust to hyperparameter choice [1, while others argue that t-SNE is not as robust to hyperparameter choice as was once believed [4, 5, 6]. Several competing hyperparameter guidelines exist [5, 1, 2, 7, but few direct comparisons have been 20 made, leaving it unclear if one has an advantage over another. Similar questions of optimal hyperparameter choices are important to other dimensionality reduction methods [6], and advances in one method could benefit another.

To address this uncertainty, we generate a large collection of t-SNE embed- 
dings of 691 data sets with a wide range of hyperparameter combinations. These embeddings show that useful ranges for t-SNE hyperparameters are narrower and include smaller values than previously reported in the literature. We also use these embeddings to empirically identify a set of hyperparameters (focusing on perplexity, exaggeration, and learning rate) that is simpler than other tSNE hyperparameter guidelines but yields more accurate embeddings, often by 30 a statistically significant margin. We perform systematic explorations of hyperparameter space to identify near-optimal hyperparameter values for each data set individually. We also use this collection to develop a neural network that guides the discovery of hyperparameters that yield accurate t-SNE embeddings for a given data set, based on its characteristics. The neural network uses as features the mean and variance of 9 graph-theoretic metrics called scagnostics [8], calculated over many random projections of the data set, plus the number of data points and number of dimensions in the data set. In our evaluation the hyperparameters predicted by our neural network yield embeddings with similar accuracy as the best current t-SNE guidelines. Importantly, we also quantify the potential for future improvements in this area: using data from a grid search of t-SNE hyperparameters we find that an optimal selection method could improve embedding accuracy by up to two percentage points over current hyperparameter selection methods. We conclude with future work to improve the neural network and explore its ability to generalize to larger data sets.

Our major contributions are:

- Guidelines for selecting t-SNE hyperparameters derived from hundreds of data sets showing that useful ranges for t-SNE hyperparameters are narrower and include smaller values than previously reported in the literature. See Section 8.1 for specific guidelines.

50

- An automatic method for identifying t-SNE hyperparameters that optimize embedding accuracy.

- Comparison of our guidelines and existing hyperparameter guidelines in a 
large study, reproducing other research and showing that t-SNE hyperparameter guidelines can be improved further.

55 2. Background and Related Work

t-Distributed Stochastic Neighbor Embedding (t-SNE) is a very popular dimensionality reduction method for visualizing high-dimensional data by "giving each point a location in a two or three-dimensional map" or embedding [1] Briefly, t-SNE tries to maximize the probability that similar points are positioned near each other in this low-dimensional map while preserving longer distance relationships as a secondary priority. Informally, t-SNE attracts points that are nearest neighbors to each other and simultaneously pushes all points away from each other. t-SNE has several hyperparameters that control these interactions. The most commonly changed are perplexity, exaggeration, and learning rate. Informally, perplexity controls the size of the neighborhood used for attracting points, exaggeration controls the magnitude of attraction, and learning rate controls the step size for the gradient descent that seeks to minimize error. Changing these hyperparameters can affect the accuracy and quality of the embedding.

\subsection{Measuring Embedding Accuracy}

Researchers typically use quality metrics to evaluate data set embeddings produced by t-SNE and other embedding methods. Bertini et al. 9 ] define a quality metric as a metric that "captures properties useful to the extraction of meaningful information about the data." Bibal and Frénay further distinguish two types of quality metrics: accuracy metrics, which quantify how the embedding preserves the data set's information, and interpretability metrics, which quantify the presence of interpretable visual patterns in the embedding [10]. An accuracy metric example is measuring the correlation between distances in the data set and the embedding; an interpretability metric example is class 
errors (by changing a point's nearest neighbors or by exaggerating distances between points) but makes it easier to distinguish the two classes. We follow Bibal and Frénay's naming convention and distinction between accuracy and interpretability metrics. Some past work has explored using quality metrics like scagnostics [8] as a proxy for human perception [1] and to identify perceptually interesting visualizations [12].

We can further distinguish accuracy metrics as being either local or global. Local accuracy metrics measure accuracy of a subset of the embedding, such as the accuracy of an individual point or a cluster, whereas global metrics measure the accuracy of the entire embedding. While local accuracy metrics can be useful for identifying and communicating individual errors, global accuracy metrics are better suited for comparing different embeddings of the same data set.

We also note that some accuracy metrics produce a scalar, but other accuracy metrics produce multi-dimensional output. Scalar metrics lend themselves to 95 easy interpretation, optimization, and integration in decision-making scenarios. On the other hand, multi-dimensional metrics provide more nuance. To simplify the automated optimization of embeddings, we focus our discussion on global accuracy metrics that produce a scalar.

In our survey of the literature, we identified the following candidate global scalar accuracy metrics on an embedding $E$ of a data set $D$ with $n$ points and $m$ dimensions (see other works for discussion of other types of metrics [6, 13, 14, 10, 15, 16, 9]):

- Root mean squared error (RMSE): The square root of the mean squared difference between the distances $d_{i j}$ in $D$ and the distances $\delta_{i j}$ in $E$ for points $p_{i}, p_{j}$ :

$$
\sqrt{\frac{2}{n(n-1)} \sum_{i=1}^{n} \sum_{j=i+1}^{n}\left(d_{i j}-\delta_{i j}\right)^{2}} .
$$

- Stress: The square root of the squared error normalised by the sum of the distances in $E$ :

$$
\sqrt{\frac{\sum_{i=1}^{n} \sum_{j=i+1}^{n}\left(d_{i j}-\delta_{i j}\right)^{2}}{\sum_{i=1}^{n} \sum_{j=i+1}^{n} \delta_{i j}}} .
$$


- Pearson: The Pearson correlation coefficient of the distances $d_{i j}$ in $D$ and the distances $\delta_{i j}$ in $E$ for points $p_{i}, p_{j}$. ness and continuity that favors locality more highly [20].

We note that there is no comprehensive guidance about which accuracy metrics to use. For example, embedding methods that emphasize neighborhood 
accuracy tend to be evaluated with neighbor-based metrics [17. But we have not seen comprehensive guidance about when to use, for example, KNN accuracy instead of trustworthiness. There are a few papers suggesting that dimensionality reduction metrics should be combined in a (weighted) average [21, 10, 22. In the related field of graph embeddings, there is some work that suggests that embeddings characterized by a high average of multiple metrics tend to be better than embeddings that maximize a single metric [23]. There is also some work relating to t-SNE that suggests that it is better to improve both neighbor-based and distance-based metrics [5].

\subsection{Identifying Good $t$-SNE Hyperparameters}

Because t-SNE has proven so popular, many researchers and software library authors have worked to identify guidelines for using t-SNE and selecting its hyperparameters [1, 5, 4, 17, 24, 2, 6]. Initially, researchers thought that tSNE was robust to hyperparameter values, in particular for perplexity [1, but gradually research showed this is not completely true [4, 6, 5, 7]. Van der Maaten and Hinton's original guideline was to set perplexity in the range 5-50. Some work on developing guidelines has been limited to specific data domains such as single-cell transcriptomics [5]. Other work has attempted to automatically derive optimal hyperparameters, which is similar to our goal. Cao and Wang [24] propose optimizing perplexity by running t-SNE on a data set multiple times and choosing the perplexity value that minimizes their objective function, which incorporates KL divergence. This requires computing multiple embeddings, which is time consuming and slows down the analysis process. This approach also violates a warning from the author of t-SNE: KL divergence should not be compared between embeddings with different perplexity values [25]. It also does not support optimizing other t-SNE hyperparameters like exaggeration or learning rate. Belkina et al. 7] suggest automatically setting the learning rate to $n / 12$, where $n$ is the number of points in the data set. They do not propose an automatic method to select perplexity because they did not find substantial evidence that it impacts the visualization. 
Although some prior work includes analysis of hyperparameter selection guidelines [6], generally prior work focuses on comparisons between embedding methods [6, 26, 16, 13, 27. This contrasts with our work, which explicitly compares hyperparameter selection guidelines for t-SNE.

\section{Data Collection}

We collected data both to develop our neural network method for identifying good t-SNE hyperparameters and to evaluate the performance of it and other tSNE hyperparameter guidelines. We collected data sets from three repositories; de-duplicated, normalized, and extracted features of the data sets; and collected accuracy metrics of data set embeddings generated by t-SNE from a grid search of hyperparameter combinations.

\subsection{Assembling Data Sets from Repositories}

We identified three common repositories of real-world tabular data sets: The UCI Machine Learning Repository [28], the R data sets package 29], and the KEEL data set repository [30. We began with all data sets from these repositories possessing at least three dimensions because in this work we create and compare two-dimensional embeddings. We removed data sets with fewer than 10 or more than 10,000 points because there were few data sets larger than this and we wanted suitable data density in order to obtain meaningful results.

After removing duplicate data sets common to multiple repositories, we were left with 791 data sets. For each of the 791 data sets we removed any nonnumerical columns. We removed rows in which any column had a non-finite value. We then normalized values within each column to have mean 0 and variance 1 , while imputing missing values to have the mean value (i.e. 0 ). We discarded data sets whose embeddings yielded errors when we attempted to calculate their scagnostics (see Section 3.3), leaving us with 691 data sets and a total of 236,496 embeddings to use in this study.

Figure 1 shows the distribution of data points and dimensions for the data sets we use in this paper, and Table 1 shows the mean and standard deviation 


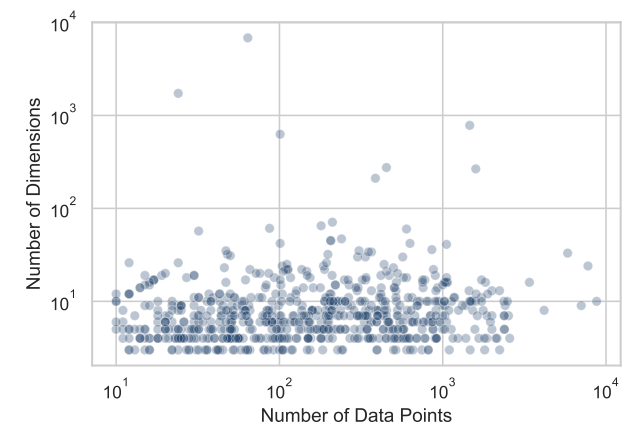

Figure 1: Scatter plot showing the distribution of the number of points and the number of dimensions of the 691 data sets we used in this paper. Each data set is one circle in this plot. Note the $\log$ axes.

of data points, dimensions, and the nine scagnostics statistics (see 3.2 for our process to calculate scagnostics). There is a skew towards data sets with fewer than 100 dimensions and fewer than 1,000 data points. As a result, this study is not applicable to the very large data sets used in domains such as image classification or bioinformatics, but our data sets represent a wide range of realworld domains for which t-SNE can be a useful dimensionality reduction and visualization technique. From the scagnostics, it appears that our data sets cover a wide range of values for some (e.g. Outlying and Convex), but others have a narrow spread centered at one end of the range of possible values (e.g. Clumpy and Sparse). This may limit how our results generalize to data sets outside these values, but because we have a large collection of real tabular data sets we expect our results to generalize well to other tabular data sets.

\subsection{Extracting Features from Data Sets}

We sought to identify characteristics fundamental to each data set for the purpose of predicting the best t-SNE hyperparameters to use in generating an embedding for that data set. To do this, it is necessary to describe the data set with summary statistics and derived features. We are not aware of prior art that attempts to featurize entire data sets for purposes such as ours, except for the cited work that used the number of dimensions or the number 


\begin{tabular}{lrr}
\hline Statistic & Mean & STD \\
\hline Data points & 407 & 750 \\
Dimensions & 24 & 271 \\
Outlying & 0.24 & 0.21 \\
Skewed & 0.62 & 0.07 \\
Clumpy & 0.08 & 0.08 \\
Sparse & 0.1 & 0.09 \\
Striated & 0.09 & 0.1 \\
Convex & 0.37 & 0.18 \\
Skinny & 0.51 & 0.11 \\
Stringy & 0.41 & 0.09 \\
Monotonic & 0.16 & 0.14 \\
\hline
\end{tabular}

Table 1: The mean and standard deviation (STD) of several descriptive statistics on our 691 data sets. See Section 3.2 for our process to calculate scagnostics on a data set.

of data points [24, 5, 7]. Therefore we propose a new method for featurizing data sets with a set of statistics intended to provide more nuance than simply the number of dimensions and the number of points. We use graph-theoretic scagnostics [8], which are a set of nine descriptive statistics all in the range [0,1]. Each of the nine scagnostics are calculated from two-dimensional data sets, and each scagnostic measures qualities of the two-dimensional data; specifically, the nine scagnostics measure how clumpy, convex, monotonic, outlying, skewed, 210 skinny, sparse, striated, and stringy the data is. Wilkinson et al. [8] describe how to calculate them, but in summary: bin the two-dimensional data in a hexagonal bin; derive a proximity graph from the binned data; calculate the minimum spanning tree, convex hull, and alpha hull geometric graphs from the binned data; and then calculate the nine scagnostics from properties of the geometric graphs. To apply scagnostics to higher dimensional data sets we derive $10+\sqrt{m}$ random two-dimensional projections of the data set and calculate scagnostics on each (where $m$ is the number of dimensions in the 
data set). Random projections are used for dimensionality reduction for $k$ means clustering [31, and a class of classification methods use multiple random projections to create an ensemble of learners 32,33 . We choose $10+\sqrt{m}$ as having a sufficient number for lower dimensional data sets while growing for higher-dimensional data sets. We compute the mean and variance of each of the nine scagnostic statistics on the $10+\sqrt{m}$ random projections. Along with the number of data points and dimensions, this yields a feature vector of 20 features for each data set. Averaging across a random sample of 138 data sets ( $20 \%$ of our data), this process takes about 1.2 seconds per data set.

\subsection{Generating Embeddings}

We used the OpenTSNE v0.3.11 implementation of t-SNE [3] to generate embeddings of the data sets. We used FFT for the negative gradient method, with exact nearest neighbors and PCA initial positions [5]. For the other hyperparameters, we used the OpenTSNE defaults, except for exaggeration, learning rate, and perplexity as described below.

We generated embeddings for each data set using a grid of t-SNE hyperparameters where exaggeration is in $\{1,2,3,4,5,6,8,16,32,64,128\}$, learning rate is in $\{10,20,40,80,160,320,640,1280\}$, and perplexity is in $\{1,2,4,8,16,32,64,128\}$ but less than $(n-1) / 3$ (because OpenTSNE does not permit perplexity greater than $(n-1) / 3)$. We chose these ranges for several reasons: the ranges are consistent with previously suggested t-SNE hyperparameter ranges; prior guidelines suggest that larger data sets benefit from larger hyperparameter values, and our data sets are smaller than the data sets used in past t-SNE studies [1, 5, 17, 17, so small hyperparameter values might yield better embeddings; and our intuition is that small differences in hyperparameter values are more important for smaller data sets than for larger data sets. We initially did not include exaggeration in $\{1,2,3,5,6\}$ and perplexity in $\{1,2\}$ but we observed that the most accurate embeddings tended to have lower exaggeration and perplexity so we generated embeddings with those hyperparameter values later (the bottom right scatterplot in Figure 3 illustrates the hyperparameter ranges we tested). 
We excluded data sets whose embeddings caused errors when we attempted to calculate their scagnostics, although we did not end up using those scagnostics.

\subsection{Measuring Embedding Accuracy}

We choose to use KNN and Spearman accuracy metrics for several reasons: t-SNE is typically evaluated this way; t-SNE is considered useful for preserving neighborhoods and also some global structure [5, 1]; and at least some distance preservation may be desired because novices may misread embeddings and presume nearby point clouds are more similar than distant point clouds. Therefore using these metrics helps make our results comparable to other work such as the OpenTSNE hyperparameter guideline, which was optimized for KNN (for $k=10)$ and Spearman accuracy [5].

However, we wish to identify how we should use these accuracy metrics. It remains unclear which $k$ to use for the KNN accuracy metric, since both $k=1$ and $k=10$ are commonly used in the literature.

We were also interested in exploring an alternative metric intended to measure rank accuracy that penalizes large changes in rank more than small changes in rank. We refer to this metric as rank accuracy. Rank accuracy is limited to the range $[0,1]$, where 1 means the distances and ranks are perfectly preserved between embedding distances and data distances, and 0 means the embedding distance ranks are in reverse order of the data distance ranks. To formally define the rank accuracy metric, let $n$ be the number of points. For a point $p_{i}$, let $\left\{r_{i, j}\right\}_{j=1}^{n}$ be the ranking of the $n$ data points by distance from 1 to $n$. So $r_{i, i}=1$ and $r_{i, k}=2$ where $p_{k}$ is the closest point to $p_{i}$ and so on. Let $r_{i, j}$ be the rankings from the original normalized data set and $\tilde{r}_{i, j}$ be the rankings from a t-SNE embedding. Then the rank accuracy metric is defined as

$$
1-\frac{1}{\Delta} \sum_{i=1}^{n} \sum_{j=1}^{n} \frac{\left|r_{i, j}-\tilde{r}_{i, j}\right|}{\min \left(r_{i, j}, \tilde{r}_{i, j}\right)}
$$

where $\Delta$ is a normalization constant defined by

$$
\Delta=\left\{\begin{array}{l}
2 n\left(n H_{n}+H_{n}-n\right) \text { and } n \text { is even } \\
2 n\left(n H_{n}+H_{n}-n+1\right) \text { and } n \text { is odd }
\end{array}\right.
$$




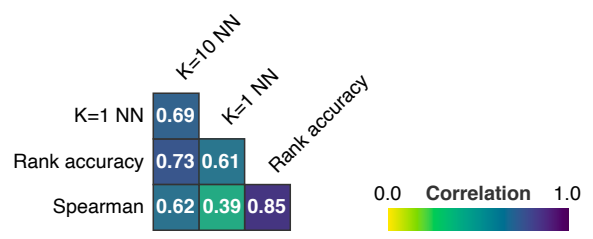

Figure 2: The Pearson correlation between the four accuracy metrics we considered in Section 3.4 for the 236,496 embeddings we generated from the 691 data sets.

for

$$
H_{n}=\sum_{k=1}^{n} \frac{1}{k}
$$

A small number of small changes to the distance ranks reduces the rank accuracy score by a small amount, and many large changes to the distance ranks reduce the score by a large amount. Rank accuracy has some similarity to Spearman correlation, but it is more analogous to an edit distance, measuring how much the distance ranking would need to change to be correct.

We exclude other metrics from our analysis for several reasons: some have seen little use in the t-SNE literature (e.g. RMSE, stress, AUC of KNN accuracy, trustworthiness, and continuity); other metrics require that data be labeled with classes (e.g. class separation and KNN class accuracy), but most of our data sets did not have explicit classes; some metrics do not align with t-SNE's stated goals (RMSE, stress) [1; others are not global metrics, such as local accuracy metrics like local continuity and local trustworthiness; and some accuracy analysis methods like the Shephard diagram and co-ranking matrix 34 do not produce scalars. And we explicitly exclude KL divergence because it should not be compared between embeddings with different perplexity values [25].

This yielded four metrics for consideration: KNN accuracy with $k=1, \mathrm{KNN}$ accuracy with $k=10$, Spearman correlation, and rank accuracy. To explore if any of the metrics are redundant, we computed the Pearson correlation between 280 these four accuracy metrics on the embeddings of the 691 data sets. Figure 2 shows the correlation matrix of these four accuracy metrics. KNN accuracy for $k=1$ and $k=10$ have moderate correlation (0.69). The KNN accuracy 
metrics are even less correlated with Spearman (0.39-0.63). Rank accuracy is strongly correlated with Spearman (0.85) and moderately correlated with the KNN accuracy metrics (0.61 and 0.73). For these reasons, we felt we should keep both of the KNN accuracy metrics. We also decided to keep the rank accuracy metric since it was not perfectly correlated with Spearman, and this provides a balance of two metrics related to distance and two metrics related to neighborhoods. There is some evidence that averaging multiple metrics can produce better embeddings in related domains [23, 21, 10, 22, and past work has argued in favor of optimizing t-SNE embeddings for both neighborhood and distance preservation [5], so we average the two KNN accuracy metrics, Spearman, and rank accuracy to produce a Mean Accuracy Metric that we use throughout this paper. We emphasize that averaging the accuracy metrics does not limit our approach for automatically tuning t-SNE hyperparameters, as we discuss in Section 5 , because users can choose to average a subset of metrics or exclude all but one metric.

We collected those four accuracy metrics and calculated them on each of the 236,496 embeddings.

\section{Data Exploration}

We can identify which t-SNE hyperparameter combination had the highest Mean Accuracy Metric for each data set using the data described in Section 3.3 and Section 3.4. We refer to this as the oracle hyperparameter guideline, because we can look up the best of all evaluated hyperparameter combinations for each data set in our collection. We also generated oracle predictions for each of the four constituent accuracy metrics separately. Although we acknowledge that there might be other hyperparameter combinations we did not test that could result in more accurate embeddings, we believe the difference in accuracy would be negligible on the tested data sets. This is because we sampled the range of hyperparameter space recommended by the literature, the top-performing hyperparameter combinations are in the region we sampled most densely (see 
analysis below), and accuracy as a function of hyperparameters does not appear to be so chaotic that a substantially better combination would exist between the combinations we sampled.

During initial data exploration, we confirmed that good t-SNE hyperparameter combinations tend to have low exaggeration and to a lesser extent low perplexity and low learning rate. Figure 3 shows a scatterplot for each pair of hyperparameters mentioned in Section 3.3. To determine each point's color, we average the Mean Accuracy Metric of the embeddings of all data sets using that hyperparameter pair and averaging across all applicable values of the third hyperparameter, then divide by the oracle's Mean Accuracy Metric for each respective data set. Darker points have embedding accuracy close to the oracle, and lighter points have worse embedding accuracy. We see the points with higher ratios tend to be hyperparameter combinations with low exaggeration.

325 The trend is less dramatic for perplexity, but we do see a similar trend where lower values tend to produce more accurate embeddings. There may also be a similar trend for learning rate. In the perplexity vs. learning rate plot the highest ratios tend to be for learning rate 10 and 20. And in the exaggeration vs. learning rate plot the ratios appear slightly lower for larger learning rates; especially when exaggeration is above 2. Points with lower average ratios often have higher standard deviation, indicating that hyperparameter combinations with lower average ratios may still produce accurate embeddings for a small number of data sets.

We also observe in Figure 3 that a relatively small number of hyperparam335 eter combinations generate the majority of the most accurate embeddings. If t-SNE users wanted to test different hyperparameter combinations to find a good embedding, the best hyperparameter combinations are most likely to have perplexity in the range $2-16$, exaggeration in the range $1-8$, and learning rate in the range $10-640$. We note that these ranges are substantially narrower and 340 include smaller values than the ranges recommended by van der Maaten and Hinton [1] and in the Scikit-learn and OpenTSNE documentation. We propose that users adopt these new ranges to guide their t-SNE hyperparameter choices 


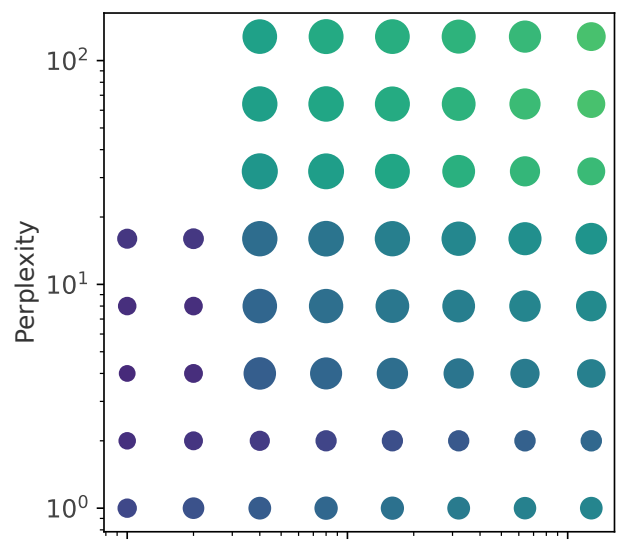

\section{Average ratio of MAM to oracle}

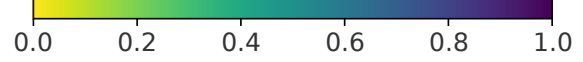

Standard deviation

(2) $\begin{array}{lll}0 & 0 & \bigcirc \\ 0.06 & 0.18 & 0.31\end{array}$
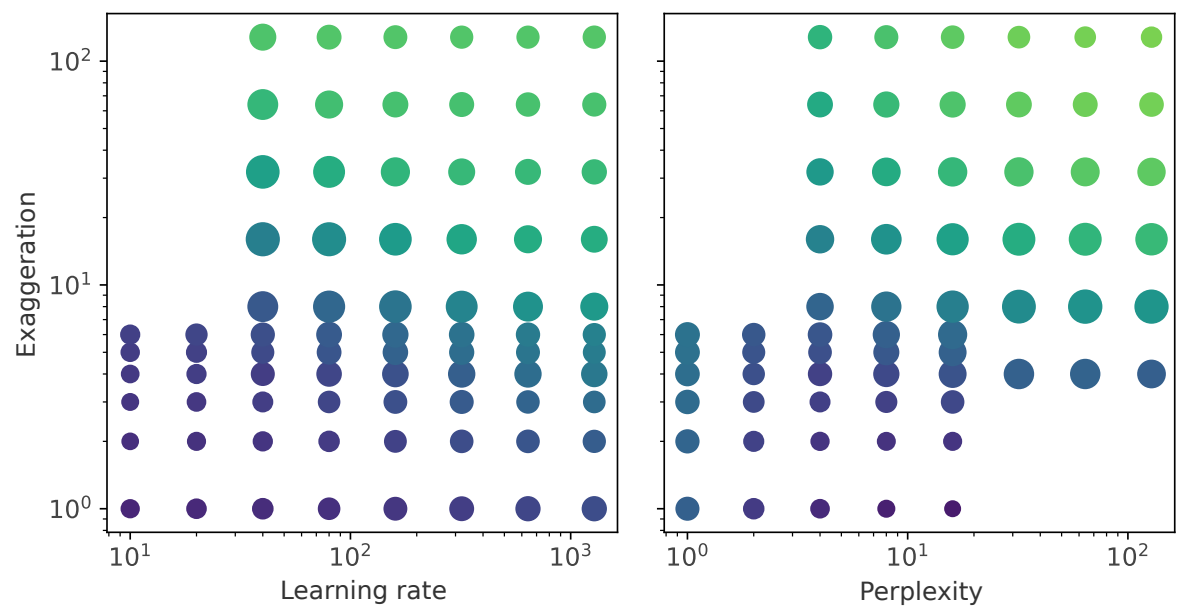

Figure 3: Average ratio of the Mean Accuracy Metric on each data set to the Mean Accuracy Metric of the data set's oracle hyperparameter combination embedding, for each tSNE hyperparameter combination. Darker hue indicates closer average accuracy to that of the oracle (i.e. the hyperparameter values generate more accurate embeddings on average), and circle size indicates standard deviation. The values for exaggeration are from $\{1,2,3,4,5,6,8,16,32,64,128\}$, learning rate from $\{10,20,40,80,160,320,640,1280\}$, and perplexity from $\{1,2,4,8,16,32,64,128\}$. Note the log scale on the axes. 
in place of the other recommended ranges, which at present suggest using perplexity in the range 5-50 and learning rate in the range 100-1000. We do note that some good hyperparameter combinations exist outside of our recommended range. If users are unsatisfied with their embeddings, they could expand the perplexity range to $1-128$, the exaggeration range to $1-16$, and the learning rate range to 10-1280; but we observed that hyperparameter combinations leading to the most accurate embeddings are less dense within these expanded ranges.

We wanted to determine whether a single hyperparameter combination could yield accurate embeddings for a wide variety of data sets. We began by identifying the hyperparameter combination that had the highest Mean Accuracy Metric for each data set. We found that the hyperparameter combination perplexity $=16$, exaggeration $=1$, and learning rate $=10$ had the highest Mean Accuracy Metric on 65 data sets, 17 more data sets than the next best hyperparameter combination. We refer to this hyperparameter combination as our empirical hyperparameter guideline. However, we also observed that the top five hyperparameter combinations all had perplexity $=16$ and exaggeration $=$ 1 ; learning rate is the only hyperparameter that changed in the five best performing hyperparameter combinations, taking on the values 20,640, 160, and 1280 in the other four hyperparameter combinations. This indicates that t-SNE is more robust to changes in learning rate around our empirical hyperparameter guideline than to changes in perplexity or exaggeration. It also demonstrates that learning rates can usefully be set smaller than 200, which was previously believed to be a good minimum value [5, 7]. This contrasts with prior beliefs about perplexity and learning rate [4, 5, 6, 1, 7.

We summarize these guidelines along with other recommendations later in Section 8.1.

\section{Tuning Hyperparameters for a Single Data Set}

We propose using a neural network to predict the best t-SNE hyperparameters to generate an embedding for a given data set. To do this, we trained 
a simple five-layer neural network. The input to the network was, for a given data set, the values of perplexity, exaggeration, and learning rate being considered, concatenated with the vector of 20 features based on graph-theoretic scagnostics 8 ] that we described in Section 3.2. The output was the four accuracy metrics we used (KNN accuracy with $k=1$, KNN accuracy with $k=10$, Spearman correlation, and rank accuracy). We trained this model using 623 of the 691 data sets we collected, reserving 68 randomly held out data sets as a test set for evaluation of the model.

In addition to an input layer of size 23 and an output layer of size 4, the neural network had three hidden layers with 40, 40, and 20 neurons, respectively. Hidden layers used rectified linear unit (ReLu) activation functions. To prevent overfitting we used $30 \%$ dropout for each hidden layer. Our loss function is the mean squared error between the predictions and the actual accuracy metrics. We trained the model using a batch size of 16 with a random $10 \%$ of the training data used as a validation set. Model training proceeded until the mean squared error on the validation set failed to decrease for more than 6 successive epochs. Then the model weights at the end of the epoch with the lowest validation error were used as the final model. We did not test other neural architecture or otherwise attempt to tune this neural network.

Table 2 shows the residual error and Pearson correlation of our model on the 68 held out test data sets on the predicted and actual accuracy metrics. These results indicate we can predict these metrics with good accuracy given scagnostics statistics for the data set and the input parameters to t-SNE.

To predict the optimal t-SNE hyperparameters for a given data set, we first iterated over all hyperparameter combinations from the earlier grid search of t-SNE embeddings and used the trained neural network to predict the four accuracy metrics for each combination. We then selected the hyperparameter combination with the highest Mean Accuracy Metric as the predicted optimum for the given data set.

Note that this process requires the neural network to predict the accuracy metrics using up to 768 hyperparameter combinations, but in practice we found 


\begin{tabular}{lrr}
\hline Accuracy metric & Residual error & Pearson correlation \\
\hline$k=1 \mathrm{NN}$ & 0.0197 & 0.876 \\
$k=10 \mathrm{NN}$ & 0.0153 & 0.859 \\
Spearman & 0.0363 & 0.725 \\
Rank Accuracy & 0.0091 & 0.803 \\
MAM & 0.0116 & 0.851 \\
\hline
\end{tabular}

Table 2: Residual error and Pearson correlation evaluation metrics on our neural network for the four individual accuracy metrics and the Mean Accuracy Metric (MAM) on the 68 held out test data sets.

that this took less than one second on our commodity laptops (in addition to the 1.2 seconds to calculate the scagnostics features, as described in Section 3.2. This is far less time than required to generate a t-SNE embedding, and therefore allows us to find approximate embedding accuracy at a significantly faster rate than generating the 768 embeddings and computing accuracy metrics on each of them. The neural network could also drive a more efficient search of hyperparameter space, not constrained by the 768 points of the grid search, which might result in both higher accuracy and faster completion. The optimum algorithm for such a search, and whether the network's interpolation and extrapolation could adequately guide such a search, are left for future work.

\section{Evaluation}

To determine performance in a range of circumstances, we assessed the Mean ${ }_{415}$ Accuracy Metric ("MAM") of embeddings produced for each of the 68 test data sets under six different hyperparameter guidelines. We show the average Mean Accuracy Metric and confidence intervals in Figure 4. The scikit-learn defaults, and the "rule of thumb" approach which combines previously published recommendations, perform significantly worse than the others.

- "Oracle" method that chooses the best hyperparameters post-hoc for each data set after the grid search described in Section 3.3. The Oracle method, 
therefore, represents the best possible hyperparameter combination in the range we tested and is the performance ceiling (over that range) for any hyperparameter selection method. Note that some methods may do better than the oracle on some data sets since they choose hyperparameters outside our tested range.

- Scikit-learn package defaults for v0.24.1, which are perplexity $=30$, no exaggeration, and learning rate $=200$ (note that we actually set it to 800 because we generate all embeddings using the OpenTSNE package, and in OpenTSNE the learning rate definition is 4 times smaller than in scikit-learn).

- OpenTSNE package defaults for v0.3.11, which are perplexity $=30$, no exaggeration, and learning rate $=\max (200, n / 12)$.

- "Rule of thumb" that combines the recommendations from past studies of t-SNE hyperparameters: Following the advice of prior work [5, we set perplexity to $1 \%$ of the data set size. In accordance with Belkina et al. [7] and Kobak and Berens [5], we set learning rate to $\max (200, n / 12)$. Finally, we fix exaggeration at 1, equivalent to no exaggeration, as is the default in the OpenTSNE and Scikit-learn implementations.

- Empirical, wherein we chose constant hyperparameter values that tended to perform well in our training data: perplexity $=16$, exaggeration $=1$, and learning rate $=10$.

- Neural Net that predicts optimal hyperparameters based on the characteristics of the data set.

We used paired $t$-tests to compare the hyperparameter selection methods with close performance. The difference in means between the empirical guideline and OpenTSNE was $0.0017(p=0.63)$. The difference in means between the empirical guideline and the neural network was $0.0087(p=0.074)$. The difference in means between the neural network and OpenTSNE was 0.0070 


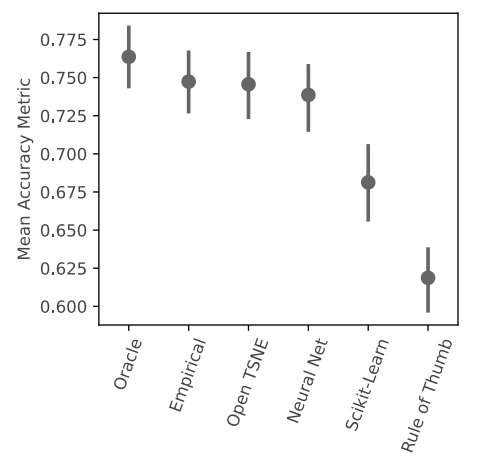

Figure 4: Mean and 95\% bootstrap confidence interval (using 1000 samples) for the Mean Accuracy Metric calculated for the embeddings produced using the six t-SNE hyperparameter guidelines for 68 data sets held out as a test group. The Mean Accuracy Metric was 0.764 for the oracle, 0.747 for our empirical guideline, 0.746 for OpenTSNE, 0.739 for the neural network, 0.681 for Scikit-learn, and 0.619 for the rule of thumb.

450 $(p=0.17)$. The empirical guideline, OpenTSNE and the neural network were all significantly better than scikit-learn and the rule of thumb $(p<1 e-4)$ and significantly worse than the oracle $(p<1 e-6)$.

We also qualitatively compare the embeddings produced using the six t-SNE hyperparameter guidelines on several data sets. Figure 5 shows two data sets from the test set (i.e. the neural network has never seen them before) and five data sets from the training set (i.e. they were used to train the neural network). In general, we observe that small differences in MAM, in the range 0.01-0.05, do not indicate obviously better embeddings. In those cases, the differences appear to reflect a varying degree of cluster separation, which is not measured by any of our metrics. The Rule of Thumb's poor performance is readily apparent in embeddings of the MedGPA, iris, and hepatitis data sets, which have substantially lower Mean Accuracy Metric.

We emphasize that averaging the accuracy metrics does not limit our approach for automatically tuning t-SNE hyperparameters, as we discuss in Sec${ }_{465}$ tion 8 , because users can choose to average a subset of metrics or exclude all but one metric. In this section and Section 5 we also demonstrate that our approach 


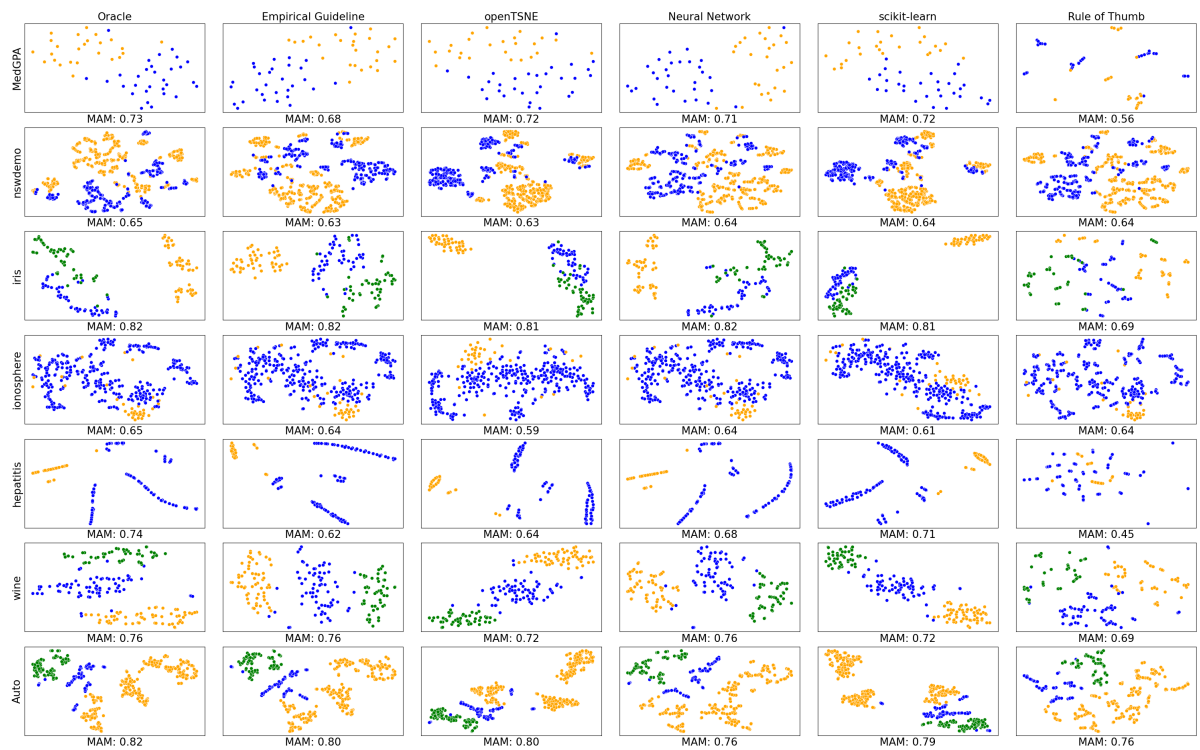

Figure 5: t-SNE embedding plots for seven data sets comparing the oracle and the five different hyperparameter guidelines. The top two rows show data sets in the neural network's test set, while the bottom five rows are in the training set. Below each embedding we show its Mean Accuracy Metric (MAM). 

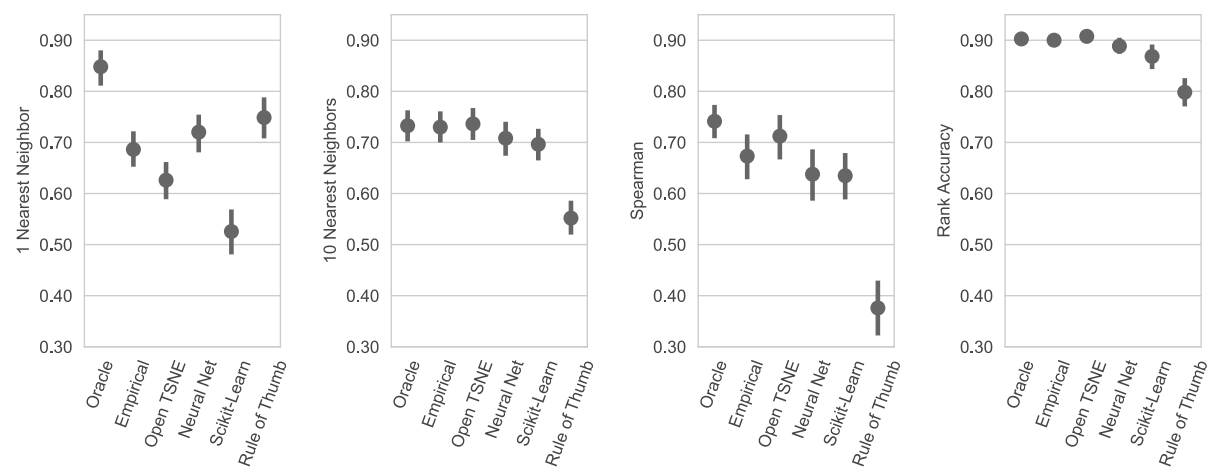

Figure 6: Mean and 95\% bootstrap confidence interval (using 1000 samples) for each of the four accuracy metrics we used, calculated for the embeddings produced using each of the six t-SNE hyperparameter guidelines for 68 data sets held out as a test group. For each of the four metrics, performance of the oracle specific to that metric (not the overall Mean Accuracy Metric oracle used in Figure 4 is shown.

to predicting accuracy metrics is successful, so if users desired embeddings optimized for different accuracy metrics we could re-train our neural network with different accuracy metrics.

We summarize these results and their implications for practitioners later in Section 8.1.

\section{Exploratory Analysis}

We explore the evaluation results in more detail in Figure 6. We observe a large difference in $k=1 \mathrm{NN}$ accuracy: The oracle is substantially more accurate than any of the other guidelines, and the neural network and rule of thumb guidelines outperform the OpenTSNE and scikit-learn defaults. On the other hand, with respect to $k=10 \mathrm{NN}$ accuracy all but the rule of thumb guideline perform very similarly. Similarly, for Spearman and rank accuracy, the two distance-based metrics, most of the guidelines perform similarly to the oracle, with the exception of the rule of thumb, which performs substantially worse.

We sought to understand if larger data sets might change the relative per- 
formance of different guidelines. We thought this might be true because the OpenTSNE guidelines were derived from studies of much larger data sets than

We also tried a different method to derive an empirical guideline. We averaged the Mean Accuracy Metric for each combination of perplexity, exagger- 

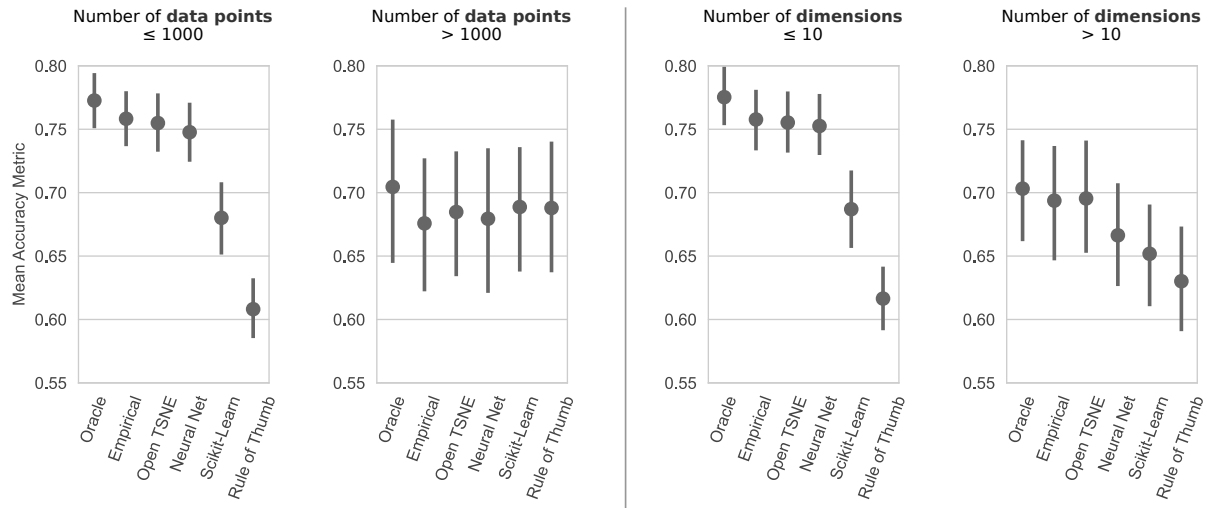

Figure 7: Mean and 95\% confidence intervals (using 1000 samples) for the Mean Accuracy Metric calculated for the embeddings produced using the six t-SNE hyperparameter guidelines for the 68 data sets held out as a test group. On the left we see the difference in Mean Accuracy Metric for data sets fewer or more data points (1,000 or fewer versus more than 1,000 data points), and on the right we see the difference in Mean Accuracy Metric for data sets with fewer or more dimensions (10 or fewer versus more than 10 dimensions).

ation, and learning rate in our grid search, then picked the hyperparameter combination with the highest Mean Accuracy Metric. This gave a hyperparameter combination of perplexity $=8$, exaggeration $=1$, learning rate $=20$. However, this combination performed minutely worse than the empirical guideline described above: its Mean Accuracy Metric on the 68 test data sets was 0.7466 compared to 0.7473 on the empirical guideline.

We summarize these results along with other recommendations for using t-SNE later in Section 8.1.

\section{Discussion}

Our evaluation provides a replication of prior work [5, 7] that indicated that setting learning rate to $\max (200, n / 12)$ achieves improved accuracy over a constant value of 800 , and consequently an improvement over the default values in Scikit-learn. This is important because prior work tested that guideline on large cytometry [7] and transcriptomics [5] data sets, and we show this guideline is effective on smaller data sets from a variety of other domains. 
However, we found that some t-SNE hyperparameter guidelines from the in Section 4, we also confirmed that the choice of perplexity does influence embedding accuracy, and we failed to find support for the recommended hyperparameter ranges from the literature and toolkit documentation [1, 2, 3]. In particular, we note that combining several t-SNE hyperparameter suggestions all other guidelines in almost all circumstances. This could be because the suggestions were derived independently and not intended to be combined as we did, which would underscore the dependence of hyperparameters on each other and the importance of deriving a holistic t-SNE guideline. Alternatively, this guideline to set perplexity to $n / 100[5]$. Our results suggest that setting learning rate to $n / 12$ is a good suggestion for both small and large data sets from many domains, but that setting perplexity to $n / 100$ is not effective for data sets of the size and type used in our study. However, we suspect that many guidelines were derived more through personal experience and less through systematic empirical analysis. A future study with a large collection of large data sets would provide additional evidence.

One reason our results may differ from early t-SNE research is that we followed the recent suggestion to use PCA to initialize positions instead of a 550 random number generator [5]. This provides more consistency between t-SNE embeddings and helps improve the accuracy of the embedding's global structure [5]. By making the embeddings less random, this might make it more obvious which hyperparameter combinations are more likely to produce accurate embeddings.

555 We believe that other embedding methods, such as UMAP [35], would benefit from a similar evaluation of hyperparameter guidelines. We also believe our general machine learning framework for picking hyperparameters can apply to other embedding techniques like UMAP, but we leave this for future work. Nonetheless, picking hyperparameters is challenging work; t-SNE was 
introduced more than 10 years ago, and the community is still exploring the best way to select good hyperparameters. For this reason, it may be preferable to develop and use embedding methods that have fewer or no hyperparameters for the user to choose.

Our motivation for using a neural network to derive t-SNE hyperparameter combinations is that it would provide high accuracy and flexibility without high complexity to t-SNE users. Users could explore t-SNE hyperparameters with the neural network to find a combination with a good Mean Accuracy Metric and in particular a high $k=1 \mathrm{NN}$ accuracy-especially compared to OpenTSNE defaults. Similarly, users could employ the neural network to find multiple hyperparameter combinations with high Mean Accuracy Metric for their data set and then explore their data through several equivalently accurate embeddings. It is possible that a different type of regression model, such as linear regression or random forest regression, would yield similar accuracy and more interpretability, but we leave this for future work. Interpretability into the neural network's predictions could be gained using a technique such as LIME [36].

Early in our research we attempted a method to directly predict good hyperparameter combinations for a data set using a decision tree. We approached this problem by finding the 5-20 most common hyperparameter combinations 50 from our grid search that maximized the Mean Accuracy Metric, then treating each combination as a class. We trained a decision tree to use a data set's scagnostics features to predict which hyperparameter combination yielded the most accurate embedding. However, on average its embeddings performed substantially worse than the neural network, so we did not pursue this approach beyond initial experiments.

Finally, we reiterate our concerns from Section 2 it is unclear which embedding accuracy metrics are best for given analysis tasks in high dimensional embeddings. Without understanding the tasks users perform and the accuracy metrics associated with successfully completing those tasks we do not have clear guidance on which accuracy metrics we should consider in work such as this. 
Tasks and embedding metrics have been well studied in the graph embedding literature $[37,38,39,40,41,42,43,44$, and we believe this is an important area for future research in low-dimensional embeddings created by t-SNE, UMAP, and related methods.

- If a balance of nearest neighbor and distance preservation is paramount, consider using the OpenTSNE defaults or our empirical guideline of perplexity $=16$, exaggeration $=1$, and learning rate $=10$ Section 6 .

- If $k=1$ nearest neighbor preservation is paramount, consider our neural network for hyperparameter selection Figure 6.

- If distance metrics are paramount (i.e. Spearman or rank accuracy) consider using OpenTSNE defaults (Figure 6), which follow some guidelines from Kobak and Berens [5] and Belkina et al. [7.

- If the above approaches yield unsatisfactory embeddings and exploration of the hyperparameter space is desired or necessary, users can follow our analysis from Section 4 explore perplexity in the range 2-16, exaggeration in the range $1-8$, and learning rate in the range $10-640$, and then if necessary expand the perplexity range to $1-128$, the exaggeration range to 1-16, and learning rate range to 10-1280 (see Figure 3). To guide this exploration, users can first try perplexity near 16,30 , or $n / 100$; exaggeration near 1 ; and learning rate near 10 or $n / 12$.

${ }^{1}$ https://scikit-learn.org/stable/modules/generated/sklearn.manifold.TSNE.html 
We suggest avoiding the default hyperparameters in the current version of Scikit-Learn (as of the time of this writing); especially on data sets with fewer than 1,000 data points or fewer than 10 dimensions Figure 7

\section{Limitations and Future Work}

We expect our results to generalize because we used real data sets from a variety of domains that we believe represent data often analyzed in practice. However, we note that all of our data sets had less than 10,000 points and dimensions (see Section 3), and we did not include some types of data sets, such as images. Therefore our results may have limited generalizability to bigger data sets or data sets from some specific domains. Future work should extend these experiments on data with more points, more dimensions, and from more domains.

We did not perform ablation testing to determine if any of the scagnostics were unhelpful features for the neural network. Future work should explore whether a subset of the scagnostics, or merely using scagnostic means instead of both means and variance, would be simpler and yield the same prediction accuracy. Similarly, we would like to explore whether $10+\sqrt{m}$ is a good number of random projections, or if a different approach would produce the same or better results.

Although we argue that our neural network is accurate enough to be used in practice, a more accurate method is likely possible because we did not test an alternative to our neural network, or attempt to optimize our neural network Section 5. Future work should attempt to improve neural network prediction accuracy with additional training data, different features (such as newer implementations of scagnostics that are more robust [45]), or tuning the neural network design and training process. There is also room for improvement even with the current training data: given an ideal architecture and training approach, our static empirical hyperparameter guideline is the floor of what a neural network should be able to achieve. 


\section{Conclusion}

We empirically derived new recommended ranges for the t-SNE hyperparameters perplexity, exaggeration, and learning rate. Interestingly, the most useful ranges are narrower and include smaller values than the ranges suggested by other researchers.

We also empirically derived a new combination of perplexity, exaggeration, and learning rate that performs as well as or better than other t-SNE hyperparameter guidelines on many real-world data sets. Interestingly, this hyperparameter combination is simpler than other guidelines that propose setting perplexity or learning rate as a function of the number of data points. This suggests that a good guideline for choosing perplexity, exaggeration, and learning rate for typical real-world data might not need any logic.

We hypothesized that we could use machine learning to predict optimally accurate t-SNE hyperparameters for any given data set, but we experienced mixed results. Our neural network produces significantly more accurate embeddings than the Scikit-learn default hyperparameters, but performs marginally worse than our empirical guideline and the OpenTSNE defaults. Tuning the neural network or improving the features could improve the embedding accuracy over our empirical guideline and the OpenTSNE defaults, but probably not by a large amount - embeddings discovered with a brute force grid search were only two percentage points better than the embeddings from our empirical guideline. This shows that there is still room to improve the accuracy of t-SNE embeddings, but probably not by very much. Larger or higher-dimensional data sets than those explored here may see larger improvements.

\section{Acknowledgments}

We thank Kevin Merchant, David Slater, and Reed Gordon-Sarney for their technical support and thoughtful discussion. 


\section{References}

[1] L. Van der Maaten, G. Hinton, Visualizing data using t-sne, Journal of machine learning research 9 (11).

[2] F. Pedregosa, G. Varoquaux, A. Gramfort, V. Michel, B. Thirion, O. Grisel, M. Blondel, P. Prettenhofer, R. Weiss, V. Dubourg, J. Vanderplas, A. Passos, D. Cournapeau, M. Brucher, M. Perrot, E. Duchesnay, Scikit-learn: Machine learning in Python, Journal of Machine Learning Research 12 (2011) 2825-2830.

[3] P. G. Poličar, M. Stražar, B. Zupan, opentsne: a modular python library for t-sne dimensionality reduction and embedding, bioRxiv preprint doi: $10.1101 / 731877$.

[4] M. Wattenberg, F. Viégas, I. Johnson, How to use t-sne effectively, Distilldoi:10.23915/distill.00002. URL http://distill.pub/2016/misread-tsne

[5] D. Kobak, P. Berens, The art of using t-sne for single-cell transcriptomics, Nature communications 10 (1) (2019) 1-14.

[6] M. Espadoto, R. M. Martins, A. Kerren, N. S. Hirata, A. C. Telea, Toward a quantitative survey of dimension reduction techniques, IEEE transactions on visualization and computer graphics 27 (3) (2019) 2153-2173.

[7] A. C. Belkina, C. O. Ciccolella, R. Anno, R. Halpert, J. Spidlen, J. E. Snyder-Cappione, Automated optimized parameters for t-distributed stochastic neighbor embedding improve visualization and analysis of large datasets, Nature communications 10 (1) (2019) 1-12.

695

[8] L. Wilkinson, A. Anand, R. Grossman, Graph-theoretic scagnostics, in: IEEE Symposium on Information Visualization (InfoVis 05), IEEE Computer Society, 2005, pp. 157-158. 
[9] E. Bertini, A. Tatu, D. Keim, Quality metrics in high-dimensional data visualization: An overview and systematization, IEEE Transactions on Visualization and Computer Graphics 17 (12) (2011) 2203-2212.

[10] A. Bibal, B. Frénay, Measuring quality and interpretability of dimensionality reduction visualizations, in: Safe Machine Learning Workshop at ICLR, 2019.

[11] A. V. Pandey, J. Krause, C. Felix, J. Boy, E. Bertini, Towards understanding human similarity perception in the analysis of large sets of scatter plots, in: Proceedings of the $2016 \mathrm{CHI}$ Conference on Human Factors in Computing Systems, 2016, pp. 3659-3669.

[12] D. J. Lehmann, S. Hundt, H. Theisel, A study on quality metrics vs. human perception: Can visual measures help us to filter visualizations of interest?, Information Technology 57 (1) (2015) 11-21.

[13] L. G. Nonato, M. Aupetit, Multidimensional projection for visual analytics: Linking techniques with distortions, tasks, and layout enrichment, IEEE Transactions on Visualization and Computer Graphics 25 (8) (2018) 26502673.

[14] M. Behrisch, M. Blumenschein, N. W. Kim, L. Shao, M. El-Assady, J. Fuchs, D. Seebacher, A. Diehl, U. Brandes, H. Pfister, et al., Quality metrics for information visualization, Computer Graphics Forum 37 (3) (2018) 625-662.

[15] B. Rieck, H. Leitte, Agreement analysis of quality measures for dimensionality reduction, in: Topological Methods in Data Analysis and Visualization, Springer, 2015, pp. 103-117.

[16] A. Gisbrecht, B. Hammer, Data visualization by nonlinear dimensionality reduction, Wiley Interdisciplinary Reviews: Data Mining and Knowledge Discovery 5 (2) (2015) 51-73. 
[21] A. Bibal, B. Frénay, Learning interpretability for visualizations using adapted cox models through a user experiment, arXiv preprint

[22] S. Johansson, J. Johansson, Interactive dimensionality reduction through user-defined combinations of quality metrics, IEEE transactions on visualization and computer graphics 15 (6) (2009) 993-1000.

[23] W. Huang, P. Eades, S.-H. Hong, C.-C. Lin, Improving force-directed graph

[25] L. v. d. Maaten, t-sne - laurens van der maaten, https://lvdmaaten. github.io/tsne/, [Online; accessed 21-August-2021] (2021). 
[26] K. Bunte, M. Biehl, B. Hammer, A general framework for dimensionalityreducing data visualization mapping, Neural Computation 24 (3) (2012) 771-804.

[27] O.-H. Kwon, T. Crnovrsanin, K.-L. Ma, What would a graph look like in this layout? a machine learning approach to large graph visualization, IEEE transactions on visualization and computer graphics 24 (1) (2017) 478-488.

[28] A. Asuncion, D. Newman, Uci machine learning repository (2007).

[29] R. C. Team, The r datasets package, R Foundation for Statistical Computing, Vienna, Austria.

[30] J. Alcalá-Fdez, A. Fernández, J. Luengo, J. Derrac, S. García, L. Sánchez, F. Herrera, Keel data-mining software tool: data set repository, integration of algorithms and experimental analysis framework., Journal of MultipleValued Logic \& Soft Computing 17.

[31] M. B. Cohen, S. Elder, C. Musco, C. Musco, M. Persu, Dimensionality reduction for k-means clustering and low rank approximation, in: Proceedings of the forty-seventh annual ACM symposium on Theory of computing, 2015, pp. 163-172.

[32] T. I. Cannings, R. J. Samworth, Random-projection ensemble classification, Journal of the Royal Statistical Society: Series B (Statistical Methodology) 79 (4) (2017) 959-1035.

[33] T. I. Cannings, Random projections: Data perturbation for classification problems, Wiley Interdisciplinary Reviews: Computational Statistics 13 (1) $775 \quad$ (2021) e1499.

[34] J. A. Lee, M. Verleysen, Quality assessment of dimensionality reduction: Rank-based criteria, Neurocomputing 72 (7-9) (2009) 1431-1443. 
[35] L. McInnes, J. Healy, J. Melville, Umap: Uniform manifold approximation and projection for dimension reduction (2020). arXiv:1802.03426.

[36] M. T. Ribeiro, S. Singh, C. Guestrin, Model-agnostic interpretability of machine learning, arXiv preprint arXiv:1606.05386.

[37] C. Dunne, S. I. Ross, B. Shneiderman, M. Martino, Readability metric feedback for aiding node-link visualization designers, IBM Journal of Research and Development 59 (2/3) (2015) 14:1-14:16.

[38] M. Pohl, M. Schmitt, S. Diehl, Comparing the Readability of Graph Layouts using Eyetracking and Task-oriented Analysis, in: Computational Aesthetics in Graphics, Visualization, and Imaging, 2009, pp. 49-56.

[39] H. C. Purchase, R. F. Cohen, M. James, Validating graph drawing aesthetics, in: International Symposium on Graph Drawing, Springer, 1995, pp. $435-446$.

[40] H. Purchase, Which aesthetic has the greatest effect on human understanding?, in: International Symposium on Graph Drawing, Springer, 1997, pp. 248-261.

[41] H. C. Purchase, The effects of graph layout, in: Proceedings 1998 Australasian Computer Human Interaction Conference. OzCHI'98 (Cat. No. 98EX234), IEEE, 1998, pp. 80-86.

[42] H. C. Purchase, D. Carrington, J.-A. Allder, Empirical evaluation of aesthetics-based graph layout, Empirical Software Engineering 7 (3) (2002) 233-255.

[43] H. C. Purchase, J.-A. Allder, D. A. Carrington, Graph layout aesthetics in uml diagrams: user preferences, J. Graph Algorithms Appl. 6 (3) (2002) $255-279$.

[44] H. C. Purchase, Metrics for graph drawing aesthetics, Journal of Visual Languages \& Computing 13 (5) (2002) 501-516. 


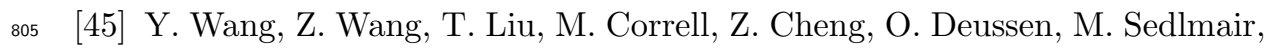
Improving the robustness of scagnostics, IEEE transactions on visualization and computer graphics 26 (1) (2019) 759-769. 